\title{
Boundary Effects on the Efficiency of Direct Infra-Red Optical Tomography
}

\author{
by J. Boulanger*, O. Balima** and A. Charette**
}

*IAR-GTL, National Research Council, Ottawa (Ontario), Canada K1A OR6, joan.boulanger@nrc-cnrc.gc.ca **AS Dept., Université du Québec à Chicoutimi, Chicoutimi (Québec), Canada G7H 2B1, Olivier_Balima@uqac.ca Andre_Charette@uqac.ca

\begin{abstract}
This study aims at emphasizing the effects of reflective boundary conditions (BC) on direct imaging with the help of simulation of radiative transfer in dense media lighted by laser beams. The direct method is based on the formation of an image whose contrast is determined by a combination of the asymptotic logarithmic regime back-scattered slopes obtained at locations distributed on the sample surface. It is found that the asymptotic logarithmic regime is strongly dependent on the frontier reflectivity. As may be expected from physics, reflecting $B C$ damage the image directly obtained as the longer path of the photons tends to blur the contrast.
\end{abstract}

\section{Introduction}

Among the new imaging modalities expected to be available in the future, optical tomography is one of the most promising. Used in industrial contexts such as flow diagnostics, substantial research is driven by biological imaging. The current applied technology is Optical Coherence Tomography $[1,2]$ and fluorescence tomography $[3,4]$. The interest in optical tomography relies on the nature of the radiation. As the name indicates, visible or infrared radiation (non ionizing) is used. Hence, equipment based on that technology is expected to be easy to handle, thus cheaper, while being harmless for the object to be probed. Low spatial resolution of this imaging method appears with high scattering properties, as in biological tissues, which makes light propagation a diffuse process (another example of the scattering effect is the sky blue colour due to intense scattering of the shortest wavelengths which are diffused when entering the atmosphere, the so-called Rayleigh diffusion).

In direct tomography, a measurable variable of the transmitted and / or reflected signals is processed in order to extract some information about the inside of a semi-transparent medium on which a laser beam has been applied. This may be the case when back-projection of the transmitted light is used along the collimated beam direction or when the apparent time-of-flight of photons is employed. In that sense, optical tomography may be compared to X-ray computer tomography [5]. In material with a high level of scattering, direct tomography is of limited use because photons do not progress on a straight line and the reconstruction is made non-linear, which prohibits the use of direct reconstruction methods such as the Radon transform. Techniques such as time-gating $[6,7]$ and spatial filtering [8] have been developed to track the less-scattered photons (the ones following as close as possible the collimated direction) but the high extinction precludes the probing of thick media. Other authors have made use of the apparent path of the photons due to the numerous scattering events through the time-of-flight of the transmitted maximum of a laser impulse [9]. The knowledge of this path is expected to provide empirical correlation for generating data but no imaging has been developed based on this method. Hence, it is unlikely that remote probing methods based on the collimated beam may be useful in a field of interest involving highly scattering semi-transparent media, like biomedical imaging. This is the reason why researchers have explored means of using the diffuse part of the signal since, in a high scattering (but low absorbing) medium, diffuse light carries not only the most energetic part of the signal but also the most informative part as well. This is due to the long time of travel that these photons spend within the medium. One of the most important features of a transient diffuse signal is its Asymptotic Logarithmic Slope (ALS) which arises long after the laser shot. Several studies made use of the ALS to determine the optical properties of some simple homogeneous media $[10,11]$. This technique has been extended to layered media [12], in the case of the application to brain oxygenation non-invasive measurement, the head being described as a multi-layered volume. Other areas are now very sensitive to this probing protocol making use of the diffuse signal as in the food industry [13], oceanography and fisheries $[14,15]$. More recently, it has been emphasized how the ALS could be used to reveal the presence of an inclusion inside a medium $[16,17]$ and numerical studies have paved the way to a potential imaging tool for direct reconstruction using the ALS diffuse signal $[18,19]$. This kind of tool for direct imaging, in the best case, is limited to structural information and the optical properties (which are a sign of inclusion identification) remain unreachable. The scattering property is related to the fine architecture of the material. If the architecture dimensions are of the order of the optical wavelength, scattering occurs [20]. Absorption is more related to the nature of the material. Another means of making use of all the information embedded in the diffuse signal is the inverse problem applied to reconstruction tomography. Much effort has been applied recently to making this imaging modality available given the new computing resources, such as clusters of P.Cs [21]. Reconstruction tomography aims at deriving quantitative images of the distribution of optical properties $\left(\sigma_{s}\right.$ and $\kappa$, respectively the scattering and absorption coefficients, both in $\mathrm{m}^{-1}$ ) of an irradiated medium given some measurements acquired at the boundary [22]. Other properties may also be obtained $[23,24]$. Rhumatoid arthritis diagnosis is a promising application $[25,26]$. Inverse problems make use of a transport model $[27,28,29]$. The experimental set-up consists of light guided by optical fibres to the surface of the sample and detecting fibres are used to collect the emerging light from the boundaries. When applied in the time-domain, several studies, [30, 31], have 
shown that filtering the data in favour of the long-term diffuse photons helps improve the quality of the reconstruction, for the same reason as mentioned before (long term photons have deeply probed the sample).

Boundary condition is not a subject frequently discussed. Some reconstructions have been done by considering boundaries, $[29,23,27]$, while others not $[32,33]$. Few publications report the consideration of boundary conditions versus imaging accuracy. Reflective boundary conditions for the diffusion approximation have been investigated [34]. For inverse reconstruction problems, it is worth noting that reflective boundary conditions are a way to enforce photons to stay longer within the sample. This has the same effects as long-term filtering, as demonstrated in [35], while being applicable to steady-state [26] or frequency-domain techniques [36, 37, 38]. In these latter approaches, time filtering is not available.

The aim of this study is to investigate the response of direct imaging with respect to reflective boundary conditions. The study is purely numerical and the algorithm is detailed in $[39,18,19]$. The first section presents the model problem. In the second section, direct imaging is explored in a way similar to $[18,19,40]$ but with the focus put on the change in the contrast of the image when reflection occurs at the boundary.

\section{Model Problem}

\subsection{Algorithm}

Light pulse propagation is of a hyperbolic nature. The time-dependent Boltzmann-type RTE given below [41, 31] is retained with usual notations:

$$
\frac{n}{c} \frac{\partial I}{\partial t}+\nabla \cdot \vec{\Omega} \cdot I+\left(\kappa+\sigma_{s}\right) I=\frac{\sigma_{s}}{4 \pi} \int_{4 \pi} d \Omega^{\prime} \Phi\left(\vec{\Omega}^{\prime}, \vec{\Omega}\right) I+\frac{\sigma_{s}}{4 \pi} \Phi\left(\vec{\Omega}^{c}, \vec{\Omega}\right) I^{c}
$$

This equation is strongly linked to the particle interpretation of light. Intensities $I$ (or $I^{c}$ ) depend on $\vec{\Omega}, \vec{\Omega}^{\prime}$ (or $\vec{\Omega}^{c}$ ), given the context. The development to the first order of the Henyey-Greenstein function is utilized for the phase function $\Phi$ [42]:

$$
\Phi\left(\vec{\Omega}^{\prime}, \vec{\Omega}\right)=1+3 g \cos \left(\vec{\Omega}^{\prime}, \vec{\Omega}\right)
$$

where $g$ is the anisotropy factor, in other words, the mean cosine of the scattered directions with respect to the incident one. The medium is non-emitting. The closure at the frontier is achieved by:

$$
I_{\cos (\widehat{\vec{\Omega}, \vec{n}})<0}=\int_{\cos \left(\widehat{\Omega^{\prime}, \vec{n}}\right)>0} d \Omega^{\prime} \rho\left(\vec{\Omega}^{\prime}, \vec{\Omega}\right) I \cos \left(\widehat{\vec{\Omega}^{\prime}, \vec{n}}\right)
$$

$I^{c}$ and $\vec{\Omega}^{c}$ are described in the next section. $\rho$ is the reflectivity and is defined later.

\subsubsection{Collimated Irradiation}

Energy enters the system through incident pulse irradiation. It is analytically integrated on its straight characteristic path $\vec{\Omega}^{c}$. Energy lost by the beam is gained by the medium through absorption and injected in the field of induced radiation intensity through scattering. This is the role of the last term in Eq. 1. The Bouguer-Beer-Lambert extinction law governs the collimated irradiation:

$$
\frac{n}{c} \frac{\partial I^{c}}{\partial t}+\nabla \cdot \vec{\Omega}^{c} \cdot I^{c}+\left(\kappa+\sigma_{s}\right) I^{c}=0
$$

Similar treatments of boundary emanating radiation have already been introduced for the homogeneous case, [43, 44, 45], to counter the ray effect. This effect arises when the discrete ordinates quadrature set does not contain all the directions in common with the boundary emanating intensity. The formal solution of Eq. 4 is:

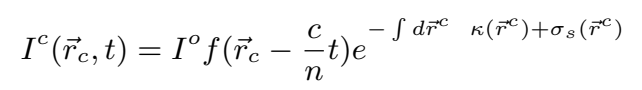

$f$ is the normalized pulse shape. $I^{o}$ is the maximum intensity. $\vec{r}^{c}=\vec{r} \cdot \vec{\Omega}^{c}$ is the distance along the beam path with respect to beam impingement location. Beam impingement is retained as the origin of the coordinate frame and time.

\subsubsection{Initial and Boundary Conditions}

No light exists in the medium before irradiation impingement. The reflectivity, $\rho$, is obtained at the boundary thanks to Fresnel-type laws as tested in [28]:

$$
\rho=\frac{\sin ^{2}\left(\alpha_{1}-\alpha_{2}\right)+\sin ^{2}\left(\alpha_{1}+\alpha_{2}\right)}{2 \cos \left(\alpha_{1}\right)}
$$


$\alpha_{1}$ is the angle with respect to the outgoing normal $\vec{n}$ of the impinging intensity from the inside of the sample. $\alpha_{2}$ is the corresponding transmitted angle in air. Both are linked by Descartes refraction laws (incident, refracted and reflected beams are all included in a plane containing the local normal to the interface $\vec{n}$ ):

$$
n \sin \left(\alpha_{1}\right)=\sin \left(\alpha_{2}\right)
$$

The refractive index of the sample is $n$ while the one of air is considered as unity. The transmitivity, $\tau$, is obtain through conservation of the intensity:

$$
\tau=1-\rho \cos \left(\alpha_{1}\right)
$$

The detectors are considered to have a full hemispherical aperture, nevertheless only the angles of emergence $(\tau>0)$ contribute to the signal. The boundary quantity that is measurable (the exitance) is defined as:

$$
d(I)=\int_{\cos \left(\widehat{\Omega_{1}, \vec{n}}\right)>0} d \Omega_{1} \tau\left(\Omega_{1}\right) I\left(\vec{\Omega}_{1}\right) \cos \left(\widehat{\vec{\Omega}_{1}, \vec{n}}\right)+\tau\left(\vec{\Omega}_{1}^{c}\right) I^{c} \cos \left(\widehat{\vec{\Omega}_{1}^{c}, \vec{n}}\right) \underbrace{}_{\cos \left(\widehat{\Omega_{1}^{c}, \vec{n}}\right)>0}
$$

This expression is written in terms of quantities inside the sample (subscript 1 ) as, because of flux conservation, the hemispherical collection of emerging intensity (i.e., for $\tau\left(\Omega_{1}\right) \neq 0$ ) is the same before and after refraction.

Equation 6 makes the assumption that a beam normal to a surface is not reflected and is fully transmitted. This assumption facilitates the analytical treatment of the collimated beam. Otherwise, the solution for an optical cavity should have been developed.

\subsection{Numerical Treatment}

The RTE (Eq. 1) is solved on a discrete set of M spatial directions covering $4 \pi$ sr solid angle. They are weighted so that space symmetry and boundary radiative balances are achieved. The discrete ordinates method has to be coupled with numerical schemes for divergence calculation and time stepping. In the following, the finite-difference method is used with, as numerical scheme, the Piecewise Parabolic Advection (PPA) TVD scheme [46, 47]. The source terms are treated implicitly and the Euler method is used for time integration. More details may be found in $[39,18]$.

The Bouguer extinction law for the collimated laser pulse is integrated with the help of a third order Runge-Kutta scheme. This enables the treatment of non-homogeneous material. Collimated and induced intensity fields are solved on collocated grids. As the CFL number is put to $1 / 2$, only one half time step over two is used to inject the total in-scattered collimated intensity into Eq. 1 corresponding to the whole time step. This partly prevents spurious progression of photons while preserving the stability of the method.

In the following sections, simulations are conducted in order to explore the effect of the boundary conditions on the emerging signal. The level of reflectivity, through the Fresnel laws, is calibrated thanks to the refractive index of the medium (the outside index is unity). The interest is on multi-dimensional simulations since, with an increase of the retention of the photons inside the material due to frontier reflexion and lower light speed, it is expected that the emerging signals are delayed with reflective boundary conditions. The main consequence is the necessity of calibrating the time span of the experiment in order to capture the backscattered ALS which constitutes the trailing part of the signal.

\section{3-D Simulations}

The sample model is a cube with two imbedded inclusions along its first diagonal at $30 \%$ and $70 \%$ of its physical length. The inclusions also have a cubic shape whose geometrical size is $20 \%$ of the whole phantom. Those inclusions differ from the background medium in terms of optical properties, namely scattering and absorption coefficients distribution.

The grid is $15^{3}$ and the quadrature set is $\mathrm{S}_{4}$. The physical time span of the experiment has been chosen such that it corresponds to twelve times the time required for light to travel across the medium. This ensures to reach the logarithmic regime and requires 180 explicit iterations (one iteration allows photons to travel from one grid node to its immediate neighbour). The top-hat pulse duration, impinging normally to boundaries, is given by $n /\left(\sigma_{s}+\kappa\right) c$ and the overall optical thickness of the cube, based on background properties (see Fig. 1a), is $\tau=10$. In Fig 1, reflectance (normalized by the laser intensity) gathered at the point of application of the laser beam (in the middle of the cube face - position (1) - and near one of its corner - position (2)) is displayed for two levels of reflection $(n=1, n=1.33)$. The detection point of the reflectance is the one of laser impingement. $t_{\text {travel }}^{*}$ is the time normalized by the delay for the light to travel across the medium.

As expected, the logarithmic slope is more pronounced in the case of unity refractive index. No reflection occurs and light easily escapes from the sample. It is also seen that for a given refractive index value, boundary effect makes the energy loss more acute when the detector is in position (2). This position is close to a corner of the cube. At that location, impingement is very near frontiers and radiations quickly escape. What is less obvious is the strong effect of reflection with respect to location of laser application. According to Fig. 1, although the laser application point may be close to a cube corner, reflection prevents the light 
(a)
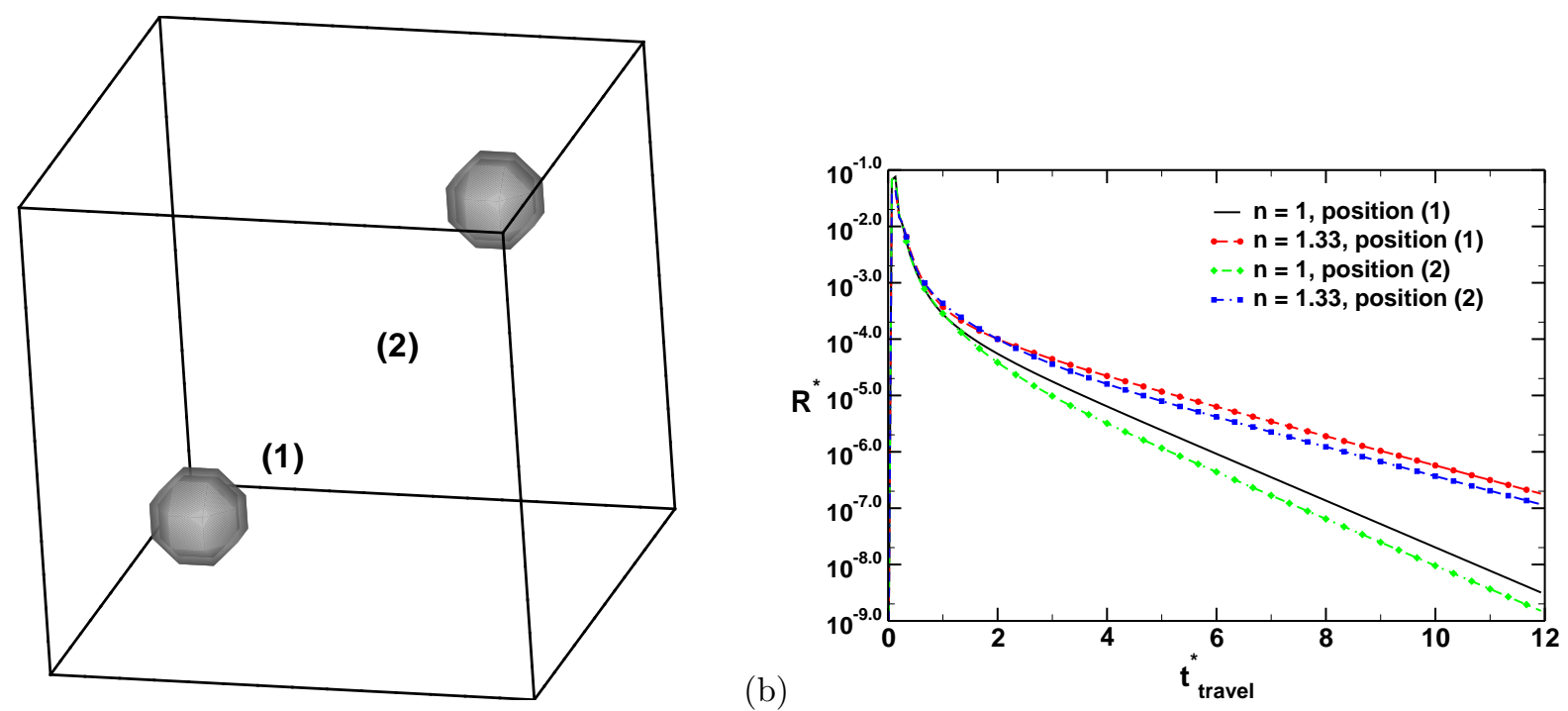

Fig. 1: Reflection levels on a 3-D medium. (a) Phantom (background: $\kappa=20 \mathrm{~m}^{-1}, \sigma_{s}=9980 \mathrm{~m}^{-1}$; top inclusion: $\kappa=15 \mathrm{~m}^{-1}$, $\sigma_{s}=13000 m^{-1}$; bottom inclusion: $\kappa=25 m^{-1}, \sigma_{s}=7000 m^{-1}$. Position (1) for pulse impingement is at the front face centre, position (2) is at the centre of the top right quarter). (b) Signature.

from escaping too much from the sample. This is to be compared to the case when the laser impinges at the centre of a face but with no reflection. This may be explained by the fact that, as the boundaries are reflecting, light entering the medium does not "see" (at least, partially) the frontier and remains in the sample such that the boundary effect appearing at the corner is weakened. For comparison, in the case of no reflection, although light is applied at the centre of a face, it escapes easily from the medium. This leads to a sharper log-slope.

Now, it is important to recall the manner by which the image is formed [18]. The imaging contrast is obtained by backprojecting the log-slope of the reflected intensity along the half-line of sight following the laser beam direction. After a complete rotation around the sample, each node within the domain has been visited by three back-propagations of log-slope magnitude. As a convention, the imaging contrast is given by the multiplication of these three values. Effects due to the finiteness of the geometry of the sample exist. A spatial filtering has to be applied to separate the contrast long scales, due to the sample shape, from the short scales, related to the inclusions presence. For ease, as it is numerically possible, use is made of the difference of the log-slopes between the sample to be probed and the homogeneous one with the same background properties.

Figures $2 a$ \& $b$ show reconstructions of the sample with and without reflection. The following comments can be made. First, the imaging contrast given by the log-slopes is reduced in the case of reflection. Second, the upper inclusion has disappeared. The underlying physical interpretation is the following: the imaging contrast is satisfactory when the log-slopes gathered on the frontier are related to the inclusion in the corresponding line-of-sight. This is at the core of this direct reconstruction methodology based on the back-scattered signal. Obviously, this relation is dramatically weakened with strong reflection. Most of the light is redistributed in the medium instead of leaving the medium when it passes a frontier. The outgoing information is thus blurred as less directly related to the immediate neighbourhood of the inclusion.

To address the contrast accuracy regarding boundary reflection properties, another direct imaging simulation has been achieved with inclusions of different natures. Instead of having "cross differences" with respect to the background properties in absorption and scattering as in the former case, both inclusions are here the same with $\kappa=15 \mathrm{~m}^{-1}$ and $\sigma_{s}=13000 \mathrm{~m}^{-1}$. The results are given in Figs 3a \& b. In that case, both inhomogeneities are retrieved, although reflection still diminishes the contrast. In fact, a general trend of contrast flattening is to be expected with reflective boundary conditions since they contribute to homogeneize the photons distribution inside the sample volume. Reflecting boundary conditions may thus be a source of reliability issues with this imaging technology.

3-D imaging simulations, being computationally very intensive, have been conducted on a Xeon processor $1 \mathrm{MB}$ cache $4 \mathrm{~GB}$ over a time span of $290 \mathrm{~h}$ each. 
(a)

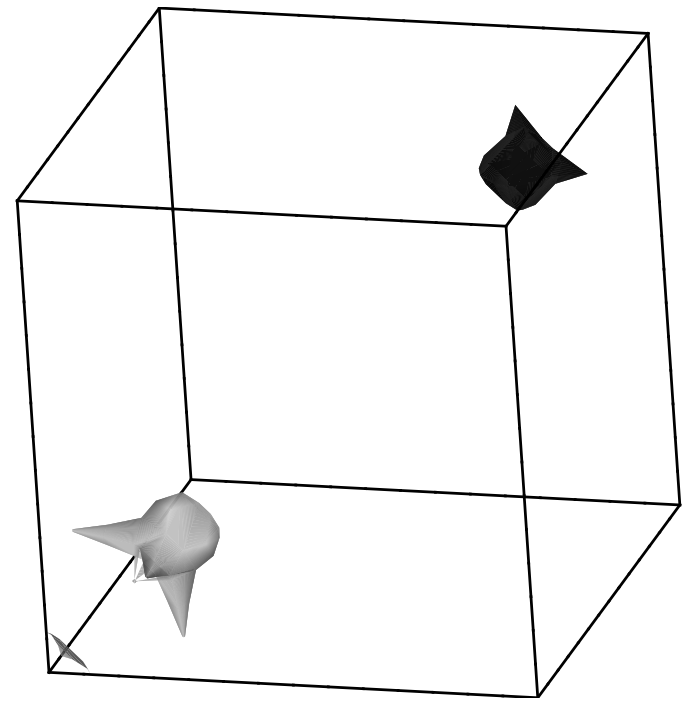

(b)

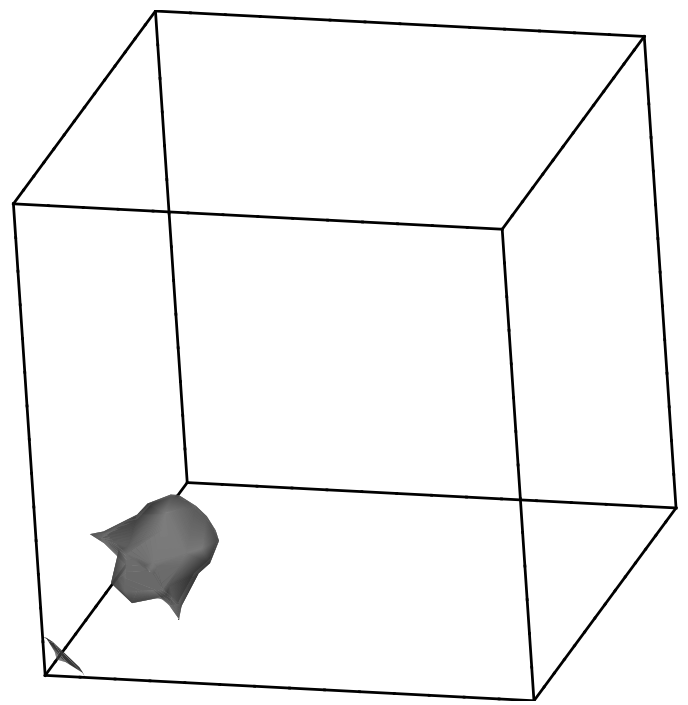

Fig. 2: Direct reconstruction. (a) Reconstruction in case of no reflection $(n=1)$, combined log-slope range: $1.84 \cdot 10^{-34}-$ $3.32 \cdot 10^{-34}$. (b) Reconstruction with reflective boundaries $(n=1.33)$, combined log-slope range: $4.57 \cdot 10^{-37}-6.85 \cdot 10^{-36}$. The log-slope values are normalized by the pulse duration. Optical properties background: $\kappa=20 \mathrm{~m}^{-1}, \sigma_{s}=9980 \mathrm{~m}^{-1}$; top inclusion: $\kappa=15 m^{-1}, \sigma_{s}=13000 m^{-1}$; bottom inclusion: $\kappa=25 m^{-1}, \sigma_{s}=7000 m^{-1}$.

(a)

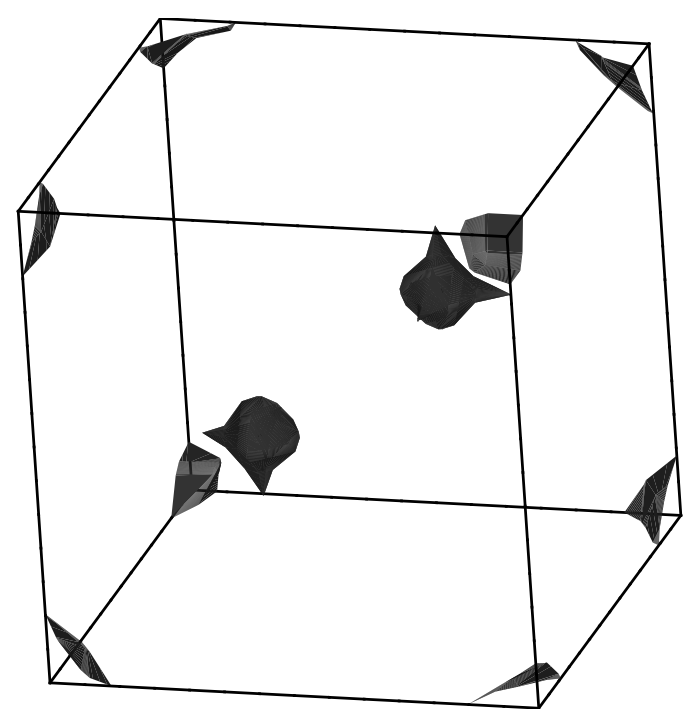

(b)

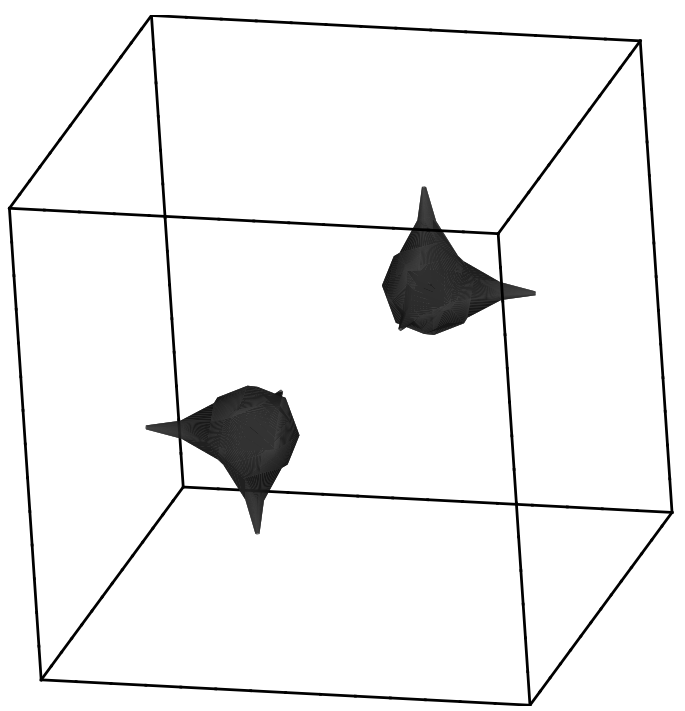

Fig. 3: Direct reconstruction. (a) Reconstruction in case of no reflection $(n=1)$, combined log-slope range: $2.15 \cdot 10^{-28}-$ $2.23 \cdot 10^{-28}$. (b) Reconstruction with reflective boundaries $(n=1.33)$, combined log-slope range: $9.19 \cdot 10^{-32}-2.02 \cdot 10^{-31}$. The log-slope values are normalized by the pulse duration. Optical properties background: $\kappa=20 \mathrm{~m}^{-1}, \sigma_{s}=9980 \mathrm{~m}^{-1} ;$ both inclusions: $\kappa=15 m^{-1}, \sigma_{s}=13000 m^{-1}$. 


\section{Conclusion}

Direct imaging does not seem to profit from reflection at the frontiers. A logical interpretation is the way the information carried by the long term photons is mixed when these latter are enforced to stay too much time in the domain. The imaging method based on the log-slope is more reliable when photons leave quickly the sample as the underlying assumption is the sensitivity of the log-slope to the closer inhomogeneities. Otherwise, reflection redistributes informative photons inside the sample with the effect of blurring the final image contrast. Back-scattered photons carrying information about an inclusion in the line of sight of the laser shot are reflected, return into the domain and are likely to leave the domain at another boundary location.

This direct imaging methodology is believed to be a fast, convenient approach to obtain structural information from a dense, scattering medium of a moderate thickness. Nevertheless, this study demonstrates that a real set-up should take care of the environment to mitigate reflection at the interface with the sample. Otherwise, the reliability may be strongly jeopardized.

\section{Acknowledgement}

Dr. William Wallace is gratefully acknowledged for his fruitful comments regarding the improvement of the manuscript.

\section{REFERENCES}

[1] Böhringer H.J., Boller D., Leppert J., Knopp U., Lankenau E., Reusche E., Hüttmann G. and Giese A., "Time-domain and spectral-domain optical coherence tomography in the analysis of brain tumor tissue," Lasers in Surgery and Medicine, 38(6), pp. 588-97, 2006.

[2] Wilder-Smith P., Jun W.G., Brenner M., Osann K., Beydoun H., Messadi D. and Chen Z., "In vivo optical coherence tomography for the diagnosis of oral malignancy," Laser Surg. Med., 33(4), pp. 269-75, 2004.

[3] Das B.B., Liu F. and Alfano R.R., "Time-resolved fluorescence and photon migration studies in biomedical and model random media," Rep. Prog. Phys., 60(2), pp. 227-92, 1997.

[4] Zavattini G., Vecchi S., Mitchell G., Weisser U., Leahy R.M., Pichler B.J., Smith D.J. and Cherry S.R., "A hyperspectral fluorescence system for 3d in vivo optical imaging," Phys. Med. Biol., 51(8), pp. 2029-43, 2006.

[5] Louis A.K., "Medical imaging: state of the art and future development," Inverse Problems, 8(5), pp. 709-38, 1992.

[6] Berg R., Jarlman O. and Svanberg S., "Medical transillumination imaging using short-pulse diode lasers," Appl. Opt., 32(4), pp. 574-79, 1993.

[7] Hebden J. and Wong K., "Time resolved optical tomography," Appl. Opt., 32(4), pp. 372-80, 1993.

[8] Wang L., Ho P. and Alfano R., "Time-resolved Fourier spectrum and imaging in highly scattering media," Appl. Opt., 32(26), pp. 5043-48, 1993.

[9] Delpy D.T., Cope M., van der Zee P., Arridge S.R., Wray S. and Wyatt J., "Estimation of optical pathlength through tissue from direct time of flight measurement," Phys. Med. Biol., 33(12), pp. 1433-42, 1988.

[10] Patterson M.S., Chance B. and Wilson B.C., "Time resolved reflectance and transmittance for the non-invasive measurement of tissue optical properties," Appl. Opt., 28(12), pp. 2331-36, 1989.

[11] Brewster M. and Yamada Y., "Optical properties of thick turbid media from picosecond time-resolved light scattering measurements," Int. J. Heat Mass Transfer, 38(14), pp. 2569-81, 1995.

[12] Hielscher A.H., Liu H., Chance B., Tittel F.K. and Jacques S.L., "Time-resolved photon emission from layered turbid media." Appl. Opt., 35(4), pp. 719-28, 1996.

[13] Valerio C., Ruiz-Altisent M., Cubeddu R., Pifferi A., Taroni P., Toricelli A., Valentini G., Johnson D. and Dover C., "Selection models for the internal quality of fruit, based on time domain laser reflectance spectroscopy," Biosystems Eng., 88(3), pp. 313-23, 2004.

[14] Churnside J.H., Wilson J.J. and Tatarskii V.V., "Lidar profiles of fish schools," Appl. Opt., 36(24), pp. 6011-20, 1997.

[15] Mitra K. and Churnside J.H., "Transient radiative transfer equation applied to oceanographic lidar," Appl. Opt., 38(6), pp. 889-95, 1999.

[16] Wan S., Guo Z., Kumar S., Aber J. and Garetz B., "Noninvasive detection of inhomogeneities in turbid media with timeresolved log-slope analysis," J. Quant. Spec. Rad. Trans., 84(4), pp. 493-500, 2004. 
[17] Guo Z., Wan S., August D., Dunn S. and Semmlow J., "Tumor imaging through temporal log-slope difference mapping of transient radiation signals," 2004 ASME International Mechanical Engineering Congress and Exposition, Anaheim, CA (USA), 2004.

[18] Boulanger J., El Akel A., Charette A. and Liu F., "Direct imaging of turbid media using long-time back-scattered photons, a numerical study," Int. J. Thermal Sc., 45(6), pp. 537-52, 2006.

[19] Boulanger J., Charette A., El Akel A. and Liu F., "Exploration de la tomographie optique directe utilisant la lumière retrodiffusée," VII ème Colloque Interuniversitaire Franco-Québécois sur la Thermique des Systèmes, Saint-Malo, France, 2005.

[20] Pham T., Spott T., Svaasand L. and Tromberg B., "Quantifying the properties of two-layered turbid media with frequencydomain diffuse reflectance," Appl. Opt., 39(25), pp. 4733-45, 2000.

[21] Hielscher A.H. and Bartel S., "Parallel programming of gradient-based iterative image reconstruction schemes for optical tomography," Comput. Meth. Prog. Biomed., 73(2), pp. 101-13, 2004.

[22] Jiang H., Paulsen K. and Österberg U., "Optical image reconstruction using DC data: simulations and experiments," Phys. Med. Biol., 41(8), pp. 1483-98, 1996.

[23] Klose A.D., Ntziachristos V. and Hielscher A.H., "The inverse source problem based on the radiative transfer equation in optical molecular imaging," J. Comput. Phys., 202(1), pp. 323-45, 2005.

[24] Khan T. and Thomas A., "Inverse problem in refractive index based optical tomography," Inverse Problems, 22(4), pp. 1121-37, 2006.

[25] Zhang Q. and Jiang H., "Three-dimensional diffuse optical imaging of hand joints: system description and phantom studies," Opt. Lasers Eng., 43(11), pp. 1237-51, 2005.

[26] Hielscher A.H., Klose A.D., Scheel A.K., Moa-Anderson B., Backhaus M., Netz U. and Beuthan J., "Sagittal laser optical tomography for imaging of rheumatoid finger joints," Phys. Med. Biol., 49(7), pp. 1147-63, 2004.

[27] Arridge S.R. and Schweiger M., "A gradient-based optimisation scheme for optical tomography," Optics Express, 2(6), pp. 213-26, 1998.

[28] Klose A.D., Netz U., Beuthan J. and Hielscher A.H., "Optical tomography using the time-independent equation of radiative transfer - part 1: forward model," J. Quant. Spec. Rad. Trans., 72(5), pp. 691-713, 2002.

[29] Klose A.D. and Hielscher A.H., "Optical tomography using the time-independent equation of radiative transfer - part 2: inverse model," J. Quant. Spec. Rad. Trans., 72(5), pp. 715-32, 2002.

[30] Schweiger M. and Arridge S.R., "Application of temporal filters to time resolved data in optical tomography," Phys. Med. Biol., 44(7), pp. 1699-1717, 1999.

[31] Arridge S.R., "Optical tomography in medical imaging," Inverse Problems, 15(2), pp. 41-93, 1999.

[32] Klose A.D. and Hielscher A.H., "Iterative reconstruction scheme for optical tomography based on the equation of radiative transfer," Med. Phys., 26(8), pp. 1698-07, 1999.

[33] Dorn O., "A transport-backtransport method for optical tomography," Inverse Problems, 14(5), pp. 1107-30, 1998.

[34] Haskell R., Svaasand L., Tsay T., Feng T., McAdams M. and Tromberg B., "Boundary conditions for the diffusion equation in radiative transfer," J. Opt. Soc. Am. A, 11(10), pp. 2727-41, 1994.

[35] Boulanger J., Liu F. and Charette A., "Investigation on the reflection at the boundaries for reconstruction laser-based imaging," J. Quant. Spec. Rad. Trans., 104(2), pp. 238-47, 2007.

[36] Kim H.K. and Charette A., "A sensitivity function-based conjugate gradient method for optical tomography with the frequencydomain equation of radiative transfer," J. Quant. Spec. Rad. Trans., 104(1), pp. 24-39, 2007.

[37] Kim H.K. and Charette A., "Frequency domain optical tomography using a conjugate gradient method without line search," J. Quant. Spec. Rad. Trans., 104(2), pp. 248-56, 2007.

[38] Abdoulaev G.D., Ren K. and Hielscher A.H., "Optical tomography as a PDE-constrained optimization problem," Inverse Problems, 21(5), pp. 1507-30, 2005.

[39] Boulanger J. and Charette A., "Numerical developments for short-pulsed near infra-red laser spectroscopy. Part I: direct treatment." J. Quant. Spec. Rad. Trans., 91(2), pp. 189-209, 2005.

[40] Boulanger J., Charette A. and Liu F., "Direct and reconstruction optical tomography. Some recent developments," Numerical Heat Transfer Conference (NHT05), Gliwice-Cracow, Poland, 2005.

[41] Arridge S.R. and Hebden J.C., "Optical imaging in medicine: II. Modelling and reconstruction," Phys. Med. Biol., 42(5), pp. 841-53, 1997. 
[42] Modest M.F., Radiative heat transfer, MacGraw-Hill, Inc., New York, 1993, ISBN 0-07-042675-9.

[43] Ramankutty M. and Crosbie A., "Modified discrete ordinates method of radiative transfer in two-dimensional rectangular enclosures," J. Quant. Spec. Rad. Trans., 57(1), pp. 107-40, 1997.

[44] Sakami M. and Charette A., "Application of a modified discrete ordinates method to two-dimensional enclosures of irregular geometry," J. Quant. Spec. Rad. Trans., 64(3), pp. 275-98, 2000.

[45] Sakami M., El Kasmi A. and Charette A., "Analysis of radiative heat transfer in complex two-dimensional enclosures with obstacles using the modified discrete ordinates method," ASME J. Heat Transfer, 123(5), pp. 892-900, 2001.

[46] Sakami M., Mitra K. and Hsu P., "Transient radiative transfer in anisotropically scattering media using monotonicity-preserving schemes," ASME International Mechanical Engineering Congress and Exposition, A. HTD-366-1, ed., Orlando, FL (USA), 2000, pp. 135-43.

[47] Colella P. and Woodward P.R., "The piecewise parabolic method (PPM) for gas-dynamical simulations," J. Comput. Phys., 54(1), pp. 174-201, 1984. 